\title{
Deformational changes after convection-enhanced delivery in the pediatric brainstem
}

\author{
Evan D. Bander, MD, ${ }^{1,2}$ Karima Tizi, MD, ${ }^{3}$ Eva Wembacher-Schroeder, BS, ${ }^{4}$ \\ Rowena Thomson, PhD, ${ }^{4}$ Maria Donzelli, MSN, ${ }^{2}$ Elizabeth Vasconcellos, MS, ${ }^{4}$ and \\ Mark M. Souweidane, MD ${ }^{1,2}$
}

\begin{abstract}
'Department of Neurological Surgery, Weill Medical College of Cornell University, New York, New York; ${ }^{2}$ Department of Neurological Surgery, Memorial Sloan Kettering Cancer Center, New York, New York; ${ }^{3}$ Department of Neurosurgery, Hôpitaux Universitaires de Genève, Geneva, Switzerland; and ${ }^{4}$ Brainlab AG, Munich, Germany
\end{abstract}

\begin{abstract}
OBJECTIVE In the brainstem, there are concerns regarding volumetric alterations following convection-enhanced delivery (CED). The relationship between distribution volume and infusion volume is predictably greater than one. Whether this translates into deformational changes and influences clinical management is unknown. As part of a trial using CED for diffuse intrinsic pontine glioma (DIPG), the authors measured treatment-related volumetric alterations in the brainstem and ventricles.
\end{abstract}

METHODS Enrolled patients underwent a single infusion of radioimmunotherapy. Between 2012 and 2019, 23 patients with volumetric pre- and postoperative day 1 (POD1) and day 30 (POD30) MRI scans were analyzed using iPlan ${ }^{\circledR}$ Flow software for semiautomated volumetric measurements of the ventricles and pontine segment of the brainstem.

RESULTS Children in the study had a mean age of 7.7 years (range 2-18 years). The mean infusion volume was 3.9 $\pm 1.7 \mathrm{ml}$ (range $0.8-8.8 \mathrm{ml}$ ). Paired t-tests demonstrated a significant increase in pontine volume immediately following infusion ( $p<0.0001)$, which trended back toward baseline by POD30 ( $p=0.046$; preoperative $27.6 \pm 8.4 \mathrm{ml}$, POD1 30.2 $\pm 9.0 \mathrm{ml}$, POD30 $29.5 \pm 9.4 \mathrm{ml})$. Lateral ventricle volume increased $(p=0.02)$ and remained elevated on POD30 $(p=$ 0.04 ; preoperative $23.5 \pm 15.4 \mathrm{ml}$, POD1 $26.3 \pm 16.0$, POD30 $28.6 \pm 21.2$ ). Infusion volume had a weak, positive correlation with pontine and lateral ventricle volume change $\left(r^{2}=0.22\right.$ and 0.27 , respectively). Four of the 23 patients had an increase in preoperative neurological deficits at POD30. No patients required shunt placement within 90 days.

CONCLUSIONS CED infusion into the brainstem correlates with immediate but self-limited deformation changes in the pons. The persistence of increased ventricular volume and no need for CSF diversion post-CED are inconsistent with obstructive hydrocephalus. Defining the degree and time course of these deformational changes can assist in the interpretation of neuroimaging along the DIPG disease continuum when CED is incorporated into the treatment algorithm. https://thejns.org/doi/abs/10.3171/2019.10.FOCUS19679

KEYWORDS convection-enhanced delivery; deformation; volumetric; hydrocephalus; diffuse intrinsic pontine glioma; brainstem

$\mathrm{C}$ ONVECTION-ENHANCED delivery (CED) is currently under investigation as a technique to bypass the blood-brain barrier and maximize drug delivery to the brain. Preclinical and initial clinical work has demonstrated that the relationship between infusion volume and distribution volume is greater than one and can be affected by a number of factors including infusion rate, cannula size, tissue substrate, and other variables. $2,4,8,10,11,14,16,21,22$ The conformational or pressure alterations that occur fol- lowing infusion of interstitial fluid during CED treatment remain poorly understood. ${ }^{1,10,21,24}$ These concerns are justifiably heightened in compact compartments such as the brainstem. Conformational changes could risk compression of important brainstem structures including cranial nerve nuclei or corticospinal tracts, could pose a risk of hydrocephalus due to compression of the fourth ventricle, and could have an influence on the interpretation of posttreatment imaging.

ABBREVIATIONS CED = convection-enhanced delivery; CTCAE = Clinical Terminology Criteria for Adverse Events; DIPG = diffuse intrinsic pontine glioma; POD = postoperative day. 
As part of an ongoing clinical trial using CED for children with diffuse intrinsic pontine glioma (DIPG) ${ }^{20}$ we sought to measure any treatment-related volumetric alterations in the human brainstem and determine if those changes had bearing on the patient's clinical condition and CSF circulation. Using a semiautomated algorithm, volumetric measurements were performed and results were correlated with preoperative findings, infusion volume, and clinical outcomes.

\section{Methods \\ Patient Selection}

Patients in this study were enrolled in a phase 1 singlecenter clinical trial using ${ }^{124} \mathrm{I}-8 \mathrm{H} 9$ monoclonal antibody ( ${ }^{124} \mathrm{I}-\mathrm{Omburtamab}, \mathrm{Y}-\mathrm{mAbs}$ Therapeutics) administered by CED. All patients were radiographically diagnosed with DIPG and were previously treated with external beam radiation therapy. The trial was registered at http:// clinicaltrials.gov (no. NCT01502917) and was approved by the IRB of the Memorial Sloan Kettering Cancer Center. Informed consent was obtained from all patients or their parents/legal guardians. Between 2012 and 2019, 39 patients were enrolled in this trial. For this retrospective review, we excluded patients below dose level 3 due to small infusion volumes $(n=7)$, patients who received multiple infusions/treatments $(n=5)$, and patients lacking volumetric pre- and postoperative imaging $(n=4)$. Twenty-three patients were included (13 girls and 10 boys, age range $3-18$ years, mean 7.7 years).

\section{Surgical Technique/Complication Grading}

The CED intervention was a single interstitial infusion of the radioimmunotherapeutic agent into the central region of the DIPG. This was performed using a supratentorial trajectory with intraprocedural MRI-guided stereotactic placement $\left(\right.$ Clearpoint $^{\circledR}$, MRI Interventions, Inc.) of a small-caliber infusion catheter (Brainlab flexible catheter, Brainlab), as previously described. ${ }^{20}$ The maximal infusion rate for all patients did not exceed $10 \mu \mathrm{l} / \mathrm{min}$. Volumes of infusion were prescribed according to the assigned dose level within the clinical trial.

An evaluation period of 30 days was used to report on treatment-related toxicity. Toxicity was assessed and graded according to the Clinical Terminology Criteria for Adverse Events (CTCAE, version 4.0). Grade 2 or greater adverse events were considered for postoperative analysis.

\section{MRI and Analysis}

For the imaging analysis, a 3D T1-weighted gradient echo brain MRI scan (slice thickness $1 \mathrm{~mm}$, matrix $256 \times$ 256 , slice spacing $0.5 \mathrm{~mm}$, field of view $22 \mathrm{~cm}$ ) was performed within 14 days prior to catheter placement, as well as on postoperative day 1 (POD1) and day 30 (POD30) after drug infusion. Data were acquired using a 3-T GE Signa scanner (GE Healthcare).

MRI scans were imported into iPlan ${ }^{\circledR}$ Flow planning software (Brainlab) for analysis (Fig. 1). Volumetric measurements of the brainstem, pons, and ventricular system were performed using the following method.

First, an automatic brainstem and ventricle segmenta- tion was applied by means of a built-in knowledge-based segmentation approach that identifies brain structures in the patient's data set and matches them to an atlas data set, in which the structures are already outlined. Using the atlas, the software finds the point-to-point correspondence of the patient's data set and the atlas data and transfers all outlined structures of the atlas into the patient data. The link between the patient's data set and the atlas data is not a rigid transformation, so that it recognizes the differences in shape and size of the anatomical structures. The atlas set is transformed elastically in such a way that the similarity of the data sets increases. Although this method has been successfully applied in adult brain segmentation, segmentation of the pediatric brain structures required modification of the automatically generated objects. Therefore, in a second step, SmartShaper, an advanced tool incorporated into iPlan for deformation of anatomical objects in three dimensions, was applied. Using SmartShaper, the user can elastically stretch and shrink contoured objects in axial, coronal, and sagittal views at one time for the intelligent update of objects in 3D space. ${ }^{5,23}$ Similar to atlas-based segmentation, SmartShaper lays a grid of invisible control points over the object to be adapted. The grid of control points can be manually pushed or pulled. As the grid is deformed, so is the corresponding object, as it is connected to the grid in all three dimensions.

In a third step, the brainstem object generated in step 2 was modified using SmartShaper to give the pons volume. For segmentation of the pons we defined the pontomesencephalic and bulbopontine sulci as the limits between the mesencephalon and the pons, and between the pons and the medulla, respectively.

For evaluation of ventricular volumes, the ventricles were separated into two objects: 1) lateral/third ventricles, and 2) fourth ventricle. Due to direct compression of the fourth ventricle, the volume of the fourth ventricle is expected to decrease after treatment. However, the lateral/ third ventricles are predicted to increase in overall volume, theoretically due to the indirect effect of diminished CSF flow through the compressed fourth ventricle. Due to these divergent volumetric changes in the lateral and fourth ventricles, assessing the ventricular system as a single object would dilute the expected changes. For parcellation of the ventricular system, an angulation of the MRI scan parallel to the line through the anterior and posterior commissures (AC-PC line) was chosen. With this angulation, we defined the upper level of the pons as the axial boundary between the lateral/third ventricle and the fourth ventricle. Using the splitting object functionality in iPlan Flow, the ventricular system object was divided along this boundary to give the lateral/third ventricle and the fourth ventricle objects. The results were verified by an experienced neurosurgeon (M.M.S.).

Volumes of each segmented object were automatically calculated and displayed by iPlan Flow. These measurements were longitudinally determined and also reported as a percentage change on POD1 and POD30 relative to the preoperative baseline value.

\section{Statistical Analysis}

Paired t-tests were used to compare preoperative and 


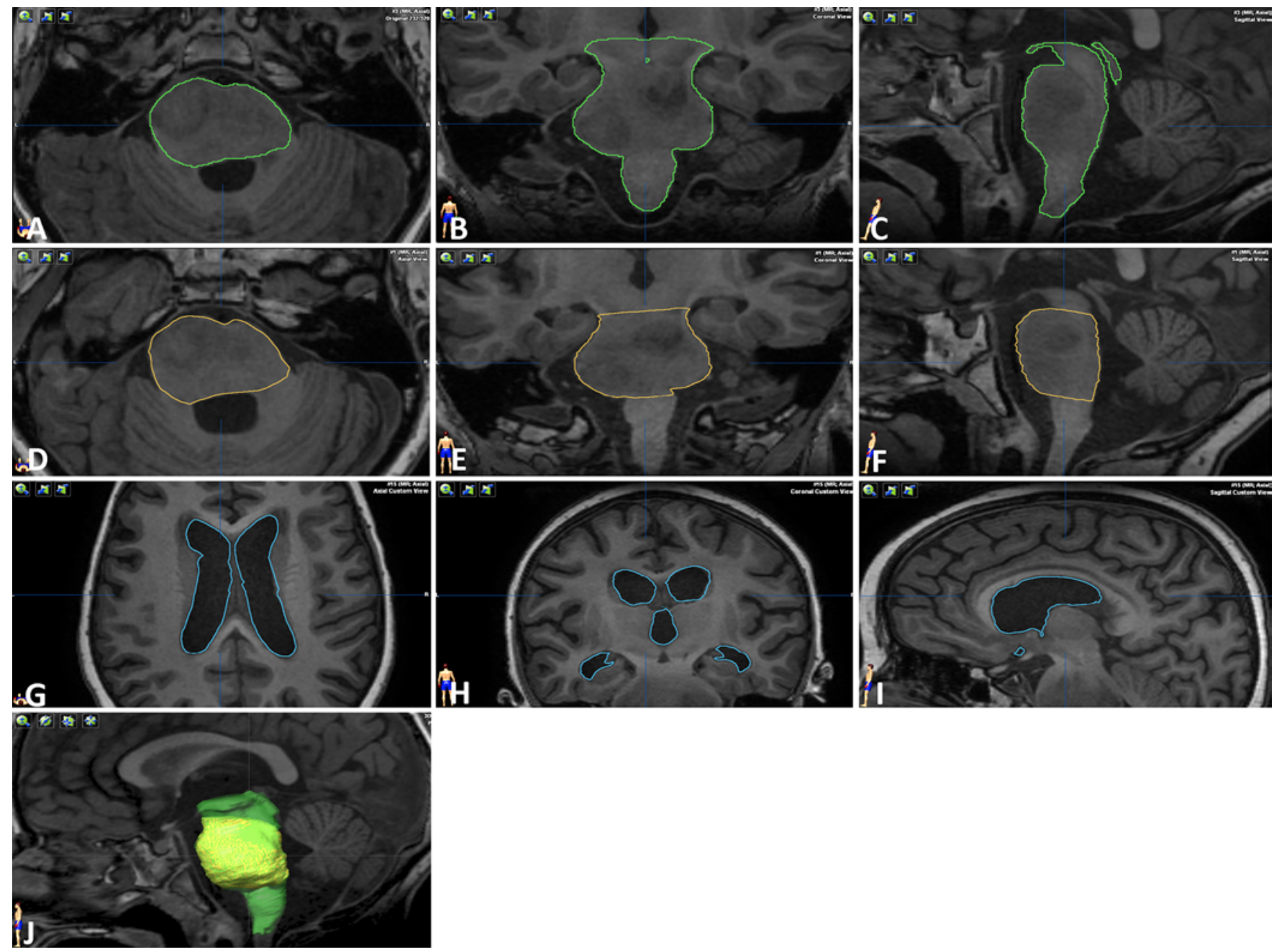

FIG. 1. MR images showing semiautomated volumetric assessment of the brainstem (A-C), pons (D-F), and ventricles (G-I), and $3 \mathrm{D}$ representation of the brainstem and pons $(\mathrm{J})$.

postoperative (POD1 or POD30) brainstem/pontine/ventricular volumes. Linear regressions were plotted and Pearson's coefficients calculated using Microsoft Excel (Microsoft Corp.). Using two-tailed t-tests, univariate analysis was performed to assess for a difference in preoperative factors of age at treatment, infusion volume, and baseline tumor volume between patients who had greater than the median or less than/equal to the median change in pontine/ventricular volume on POD1. Correlation of tumor volume and change in pontine or ventricular volume was restricted to a group of patients at dose levels 7, 7.1 , and 7.2, all of whom had relatively constant infusion volumes (range $3.8-4.5 \mathrm{ml}$ ), although escalating infusion rates $(n=13)$. A p value $<0.05$ was considered statistically significant.

\section{Results}

\section{Demographics}

Twenty-three patients were included in this study (13 girls, 10 boys). The ages ranged from 3 to 18 years (mean 7.7 years). The mean CED infusion volume for the cohort was $3.9 \pm 1.7 \mathrm{ml}$ (range $0.8-8.8 \mathrm{ml}$ ). Twelve patients had preoperative neurological deficits, while the remaining 11 were neurologically intact at baseline. Preoperative volumetric data are displayed in Table 1. There was no statistical difference in baseline tumor volume in patients with baseline neurological deficits compared to those without baseline deficits $(\mathrm{p}=0.21)$.

\section{Deformation of Brainstem and Ventricular Change}

Figures 2 and 3 demonstrate individual patients' brainstem (Fig. 2, left), pontine (Fig. 2, right), lateral ventricle (Fig. 3, left), and fourth ventricle (Fig. 3, right) volumes over time (before CED, at POD1, and at POD30).

Compared to preoperatively, on POD1 there was a statistically significant increase in the volume of the brainstem (preoperative $39.5 \pm 9.9 \mathrm{ml}$, POD1 $41.9 \pm 9.9 \mathrm{ml}$; < 0.0001 ) and pons (preoperative $27.6 \pm 8.4 \mathrm{ml}$, POD1 30.2 $\pm 9.0 \mathrm{ml} ; \mathrm{p}<0.0001)$. By POD30, the volume of the brainstem compared to preoperatively was no longer significantly higher (POD30 $41.3 \pm 10.9 \mathrm{ml} ; \mathrm{p}=0.12$ ), and while the pons remained statistically different, that difference 
TABLE 1. Demographics of study population

\begin{tabular}{lrrrrrr}
\hline & & \multirow{2}{*}{$\begin{array}{c}\text { CED } \\
\text { Variable }\end{array}$} & \multicolumn{5}{c}{ Pge } & Infusion & \multicolumn{4}{c}{ Preop Vol (ml) } \\
\cline { 5 - 7 } (yrs) & Vol (ml) & Tumor & Brainstem & Ventricular & Pontine \\
\hline Average & 7.7 & 3.9 & 19.9 & 39.5 & 24.6 & 27.6 \\
\hline Min & 3.6 & 0.8 & 6.0 & 26.0 & 8.7 & 15.1 \\
\hline Max & 18.0 & 8.8 & 48.7 & 67.0 & 67.5 & 50.1 \\
\hline SD & 3.7 & 1.7 & 11.3 & 9.9 & 14.7 & 8.4 \\
\hline
\end{tabular}

had decreased (POD30 $29.5 \pm 9.4 \mathrm{ml} ; \mathrm{p}=0.046)$. While the brainstem and pontine volume changes on POD1 compared to preoperatively demonstrated a moderate correlation $\left(r^{2}=0.63\right)$, the percentage volume change in the pons was significantly larger than that of the brainstem volume change $(p=0.0005)$. This suggests that pontine volume change is a more sensitive indicator for effects of CED than brainstem volume change.

Ventricular volume was assessed at the level of both the direct, compressive effect of CED on the infratentorial fourth ventricle, and the more global effect of the CED treatment on the supratentorial lateral/third ventricle size. The change in volume of the fourth ventricle trended toward significance on POD1 $(p=0.065)$ and was not significant by POD30 ( $\mathrm{p}=0.25)$. In contrast, the lateral ventricles demonstrated a significant increase in volume POD1 $(\mathrm{p}=0.02)$, and this remained statistically significant on POD30 compared to preoperatively ( $\mathrm{p}=0.04$; preop- erative $23.5 \pm 15.4 \mathrm{ml}$, POD1 $26.3 \pm 16.0 \mathrm{ml}$, POD30 28.6 $\pm 21.2 \mathrm{ml}$ ).

\section{Infusion Volume and Immediate Conformational Changes}

Linear regression analysis demonstrated a weak, positive correlation of infusion volume with percentage change in pontine volume on POD1 $\left(\mathrm{R}^{2}=0.22\right)$. A similarly weak, positive correlation existed between infusion volume and change in lateral ventricle volume on POD1 $\left(\mathrm{R}^{2}=0.27\right.$; Fig. 4). However, the change in pontine or ventricle size on POD30 compared to preoperatively had a negligible correlation with infusion size $\left(\mathrm{R}^{2}=0.01\right.$ and 0.07 , respectively).

The percentage change in pontine volume on POD1 demonstrated a weak, positive correlation with percentage change in the lateral ventricle volume on POD1 $\left(\mathrm{R}^{2}\right.$ $=0.26$ ). Furthermore, while infusion volume did not correlate significantly with POD30 change in lateral ventricle volume, there was a moderate, positive correlation between POD30 lateral ventricle volume percentage change and POD1 percentage change in the lateral ventricle $\left(\mathrm{R}^{2}\right.$ $=0.59)$, and weak positive correlations with percentage change in pontine volume on POD1 $\left(\mathrm{R}^{2}=0.20\right)$ and POD30 ( $\mathrm{R}^{2}=0.40$; Fig. 5).

Univariate analysis was performed to assess factors predictive of patients who had greater than the median percentage change in pontine or ventricular volume on POD1 (Table 2). We found a statistically significant difference in infusion volume of patients with greater than the median percentage change in pontine volume compared to those with less than or equal to the median change in pontine

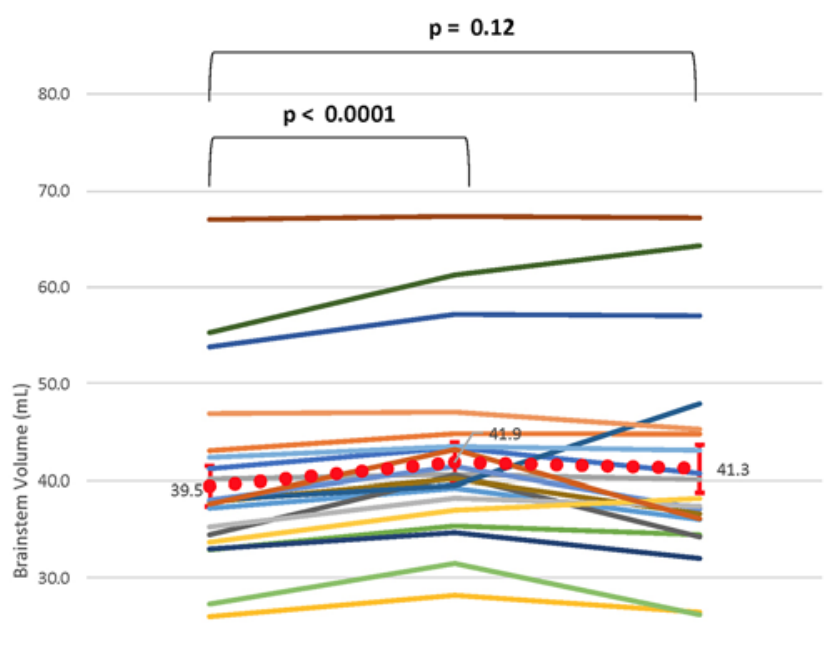

20.0

10.0

0.0
PreCED POD1 (3D T1)

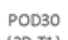

(30 T1)

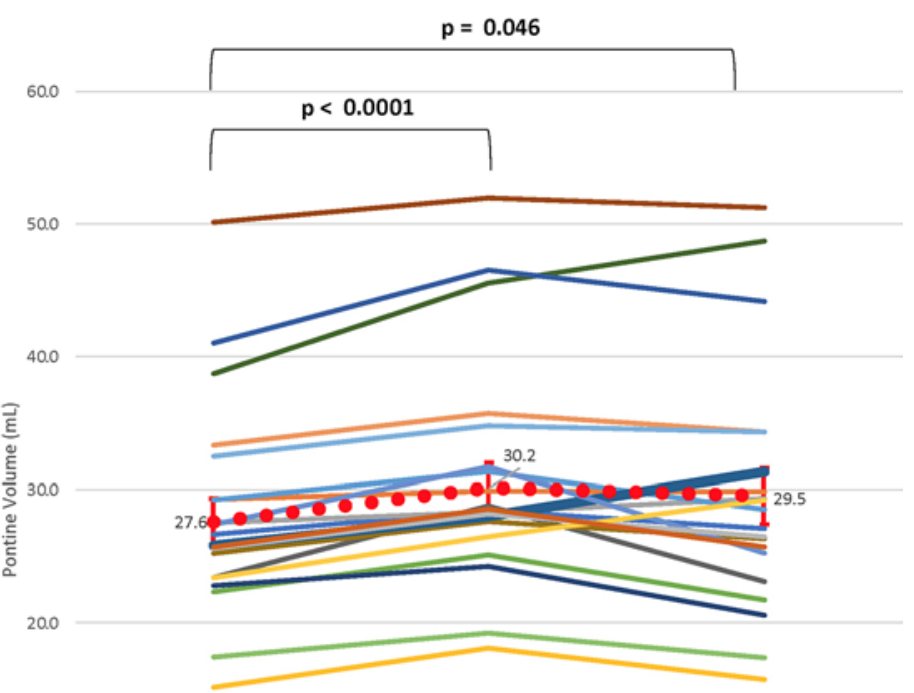

10.0

0.0

FIG. 2. Brainstem (left) and pontine (right) volume change over the course of treatment. The dotted red line represents the mean for each time point in the cohort, with red error bars representing the standard error of the mean (SEM). The $p$ values were calculated using the paired t-test. 

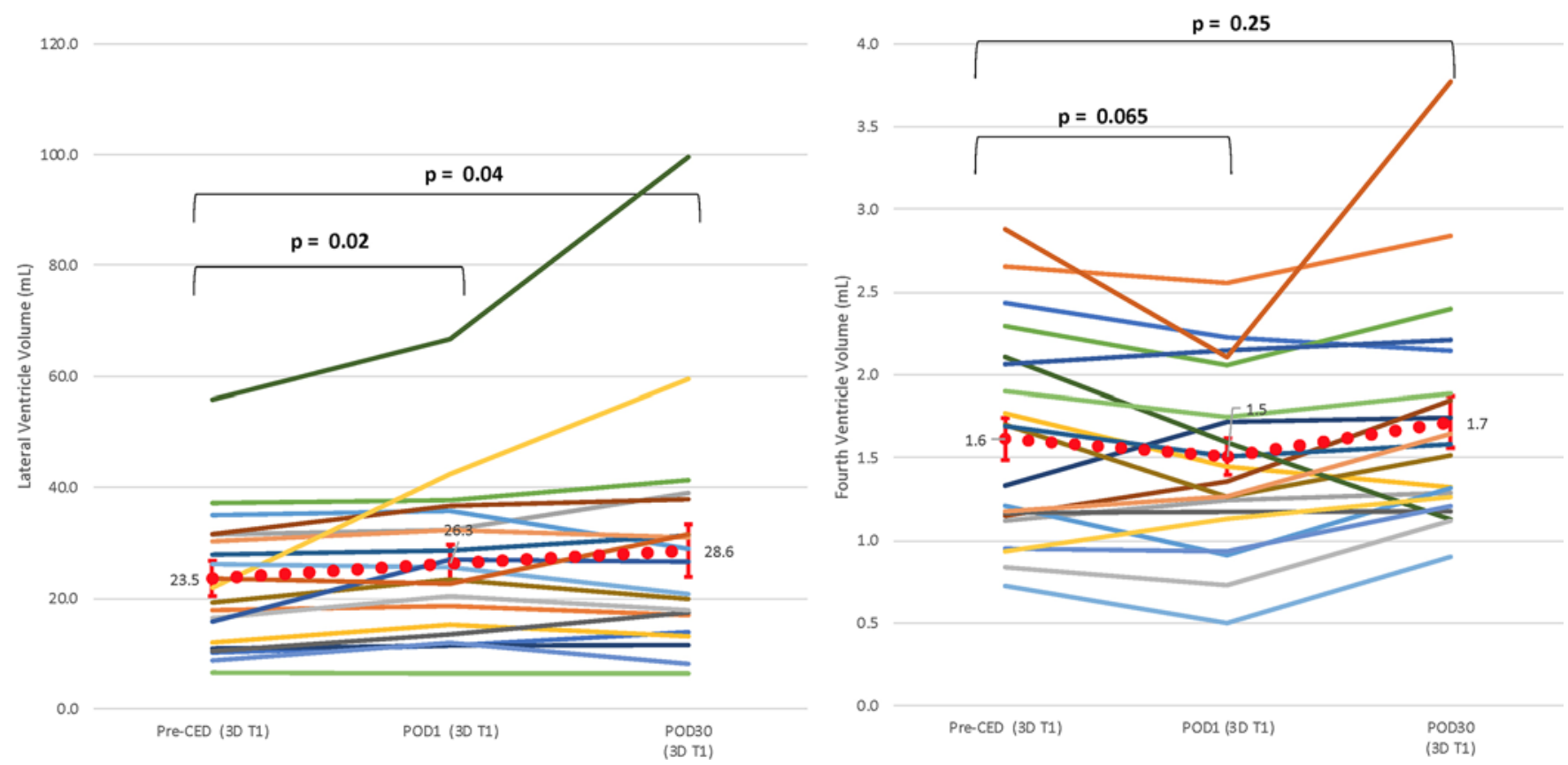

FIG. 3. Lateral (left) and fourth (right) ventricle volume change over the course of treatment. The dotted red line represents the mean for each time point in the cohort, with red error bars representing the SEM. The $p$ values were calculated using the paired t-test.

volume $(p=0.009)$. There was no significant difference in age between these two groups ( $p=0.086)$, nor baseline tumor volume $(p=0.18)$. For change in ventricular size, patient age, infusion volume, and tumor volume were not significantly different in patients with greater than the median percentage change in ventricular volume compared to those with less than or equal to the median ventricular volume change ( $\mathrm{p}=0.65,0.084$, and 0.13 , respectively).

\section{Preoperative Tumor Volume and Deformational Changes}

Preoperative tumor volume demonstrated a weak, positive correlation with preoperative lateral ventricle volume $\left(\mathrm{R}^{2}=0.27\right)$. However, when controlling for infusion volume by examining only patients with a similar infusion volume, the preoperative tumor volume demonstrated neg- ligible correlation with POD1 percentage change in pontine $\left(\mathrm{R}^{2}=0.09\right)$ or lateral ventricle $\left(\mathrm{R}^{2}=0.005\right)$ volume. These correlations also remained negligible when including the entire cohort for analysis.

\section{Clinical Outcomes}

In general, the CED infusions were tolerated without long-term neurological compromise. In a subset of patients $(n=6)$, CTCAE grade 2 or greater motor/cranial nerve findings, such as hemiparesis, facial nerve palsy, or dysarthria/dysphagia, were observed on POD1. On POD30, 4 of those patients $(17 \%)$ still had grade 2 or greater worsened neurological status (i.e., increased hemiparesis or cranial nerve palsy) compared to pretreatment baseline. However, patients with preoperative neurological deficits tended
POD1 Pontine Volume \% change vs Infusion Volume

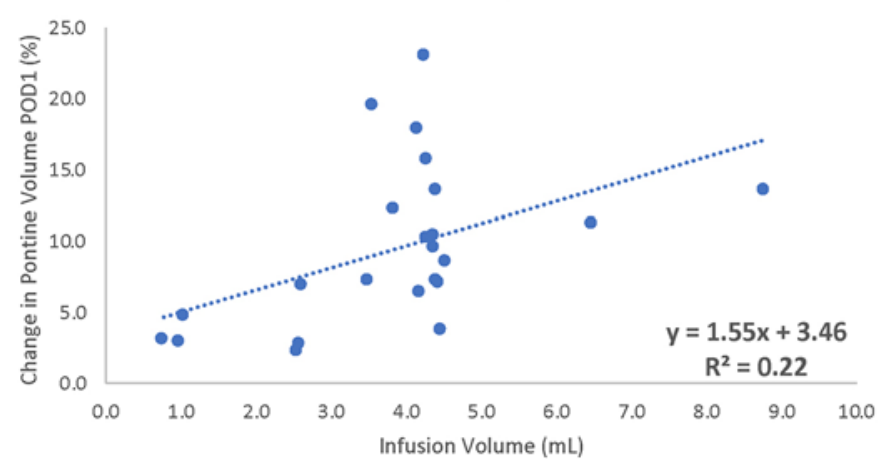

POD1 Lateral Ventricle Volume \% change vs Infusion Volume

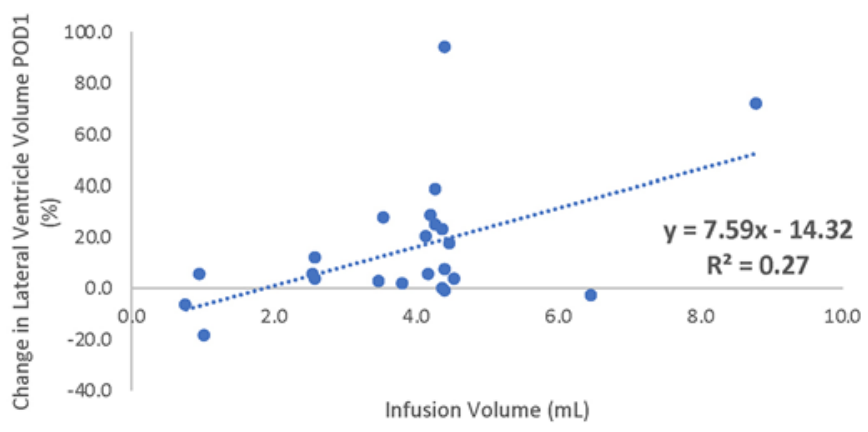

FIG. 4. Infusion volume demonstrated weak, positive correlations with change in pontine (left) and lateral ventricle (right) volume on POD1. 
POD1 Pontine vs POD1 Lateral Ventricular volume change

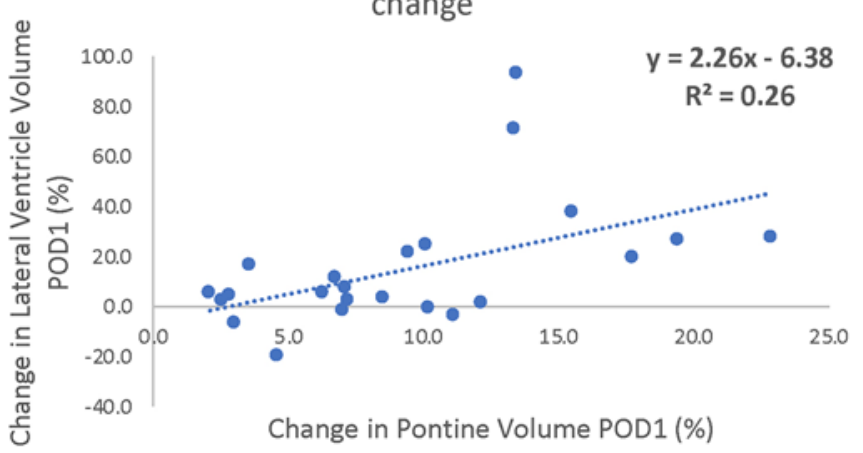

POD1 Pontine vs POD30 Lateral Ventricle volume

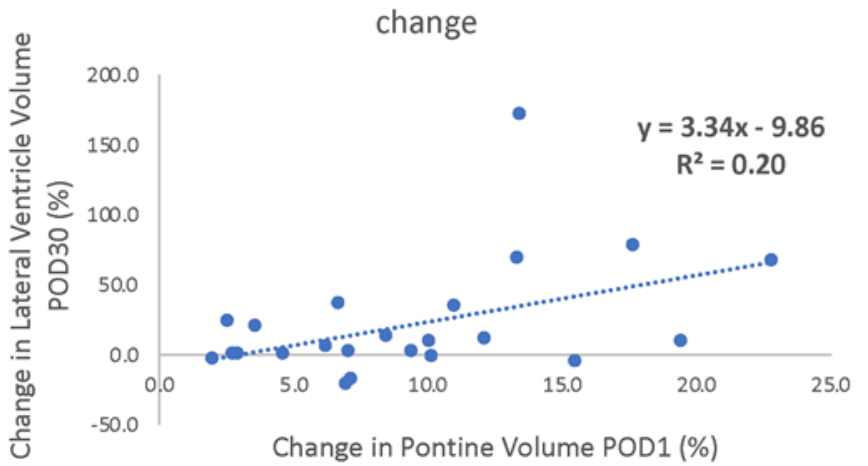

POD30 Pontine vs POD30 Lateral Ventricle volume change

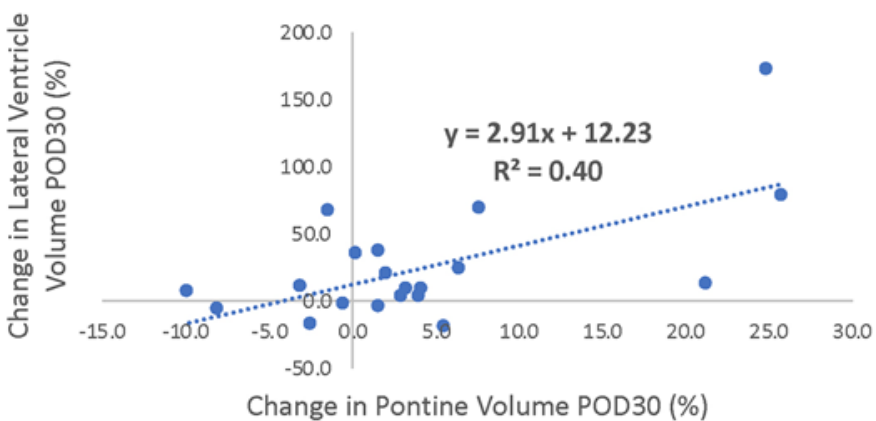

POD1 Lateral Ventricle vs POD30 Lateral Ventricle volume change

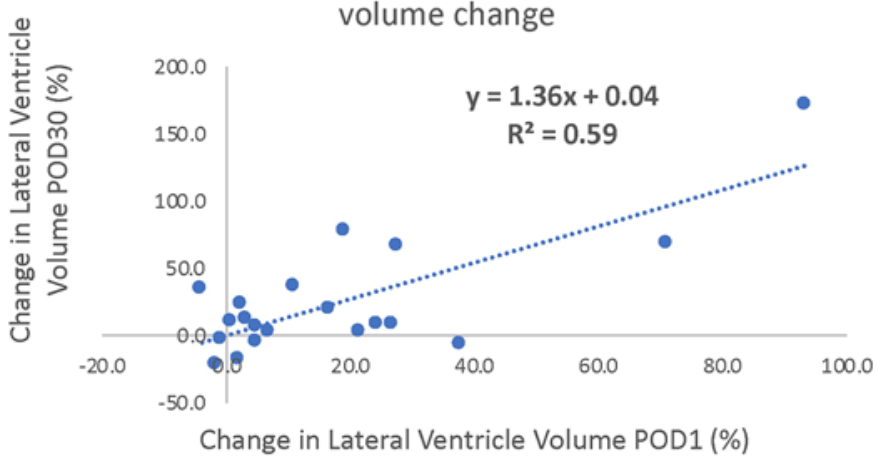

FIG. 5. Lateral ventricle volume change demonstrated positive correlations with pontine volume change at POD1 and POD30 as well as between POD1 and POD30 lateral ventricle volume change.

to have a higher risk of developing these post-CED deficits. All 4 patients with worsened neurological status on POD30 had these deficits at baseline but to a lesser degree $(4 / 12,33 \%)$, while patients who had no baseline deficits never developed neurological deficits post-CED in this cohort $(0 / 11,0 \% ; \mathrm{p}=0.09)$.

The percentage change in pontine volume did not demonstrate a significant relationship to postoperative deficits. On POD1, patients with pontine volume changes below the median had a higher proportion of neurological deficits than those with pontine volume changes greater than the median (4/13 [30\%] vs 2/10 [20\%]; $p=0.66)$. Patients with increased pontine size on POD30 compared to baseline also did not demonstrate a statistically significantly higher proportion of postoperative deficits than patients with a decreased pontine size compared to preoperative baseline ( $28.6 \%$ vs $0 \%, p=0.268)$.

Headaches occurred in 8 patients (34\%). No patients had symptomatic elevated intracranial pressure that necessitated medical or surgical treatment. No patients required shunt placement within 90 days following the CED procedure.

\section{Discussion}

DIPG represents the most common form of brainstem glioma in children and carries a dismal prognosis. The median survival duration for children with DIPGs is less than 12 months, despite decades of clinical trial development. ${ }^{3}$
Efficient drug delivery to the target tissue is commonly cited as a therapeutic obstacle in primary tumors of the brain and brainstem. CED is one form of direct drug delivery that bypasses the blood-brain barrier and potentiates drug concentration in the site of interest while avoiding systemic exposure. Preclinical studies using CED paved the way for clinical introduction. $2,4,7,10,11,14,16-19,24-26$ Limited clinical experience led to development of a systematic clinical trial aimed at assessing the tolerance of CED in the brainstem, which has now validated the infusion parameters and theoretical principles in children with DIPG. ${ }^{20}$

Studies in nonhuman primates and canines with brain tumors employing CED in the supratentorial compartments have demonstrated detectable but transient regional increases in pressure with compression of the lateral ventricles. ${ }^{21}$ These changes have not been characterized in the human brainstem. Furthermore, due to the more compact nature of the brainstem, these types of alterations might be less well tolerated. The objective of this study was to characterize conformational alterations and effects on clinical outcome and ventricular flow after CED in the human brainstem. Our findings as they relate to pontine volumetric changes are logical. Some proportional increase in brainstem size per infused unit volume was anticipated. This relationship, however, had not been previously characterized. The volumetric expansion of the pontine segment of the brainstem was also logically believed to impart physical stresses on the fourth ventricle with possible 
TABLE 2. Univariate analysis of factors related to greater than the median change in pontine/lateral ventricular volume on POD1

\begin{tabular}{lcc}
\multicolumn{1}{c}{ Factor } & Pontine Change & Lateral Ventricular Change \\
\hline Age & 0.086 & 0.648 \\
\hline Infusion vol & $0.009^{*}$ & 0.084 \\
\hline Preop tumor vol & 0.180 & 0.128 \\
\hline
\end{tabular}

The $p$ values were calculated using a two-tailed t-test.

* Statistically significant difference $(p<0.05)$.

interruption of CSF circulatory pathways. Our results suggest that this is also, in fact, true.

The relevance of our findings is threefold. First, it provides clarity in the interpretation of MRI scans performed shortly after CED. Specifically, increases in brainstem volume detected on postinfusion imaging can be more confidently attributed to treatment effect and not disease progression. Other imaging features including the degree of contrast enhancement or new exophytic components, which were not systematically reviewed in our study, might be important in distinguishing between expected infusion-related brainstem increases and disease progression. Furthermore, understanding the dynamic readjustments in brainstem volume with more frequent data points will be important in interpreting the timeline of disease response or progression. The return toward baseline in brainstem volume does support the feasibility to use serial, repeat infusions. ${ }^{9,12,15}$ However, multiple factors will play a role in the safety of repeat infusions, and the timeline for these repeat infusions remains unclear. The relationship we detailed between infusion volume and pontine/ventricular changes suggests a need for caution with continuous large-volume infusions within the brainstem. There remains a concern that large-volume, continuous infusion could cause large conformation/pressure changes, with no clear plateau in these changes yet identified.

A second meaningful finding was the consistent but modest increase in the ventricular size, presumably resulting from some diminution of the fourth-ventricle compartment. Notably, ventricular volumes did not show as much resolution compared with brainstem volumes at 30 days. This could be related to continued pressure changes interrupting ventricular flow, as there was some correlation between patients with persistent pontine volume increase and ventricular volume increase on POD30. However, this phenomenon may also be explained by a more protracted return to baseline or variations in patient-specific ventricular compliance, or may be secondary to general treatment-related volume loss. Alternatively, this change in ventricular size may be unrelated to the treatment intervention. Use of steroid therapy or non-CED treatment-related changes could affect a diminution of cerebral parenchymal volume. This possibility is somewhat supported by the lack of hydrocephalus symptoms and the persistence of ventriculomegaly in the face of the brainstem volumes returning to baseline. Regardless of the cause, the persistent changes described should be monitored in patient followup. Beyond POD30, we cannot comment on volumetric changes due to lack of standardized imaging in the trial beyond that time point. Based on the lack of symptomatic hydrocephalus in our cohort, there is no need for pre-CED shunting and the decision to place a shunt should continue to be based on clinical factors as opposed to ventricular volume changes.

Lastly, posttreatment parenchymal and ventricular volumetric changes were more apparent when the analysis was limited to the pons compared with the entire brainstem. In addition to the pons being the site of infusion, there is also reduced interstitial fluid within the tumor that would impart a greater expansion per unit volume of infusate compared with unaffected parenchyma. This result dissuades the use of automated imaging algorithms based on predetermined aggregate components of the brainstem including the mesencephalon, metencephalon, and myelencephalon. Therefore, the use of a manual adjustment tool or the creation of a more defined pontine algorithm for patients undergoing brainstem CED is needed to enhance the detection of anatomical changes. Our findings paradoxically demonstrate that larger volume changes in the pons did not relate significantly with higher likelihood/ occurrence of neurological complications, supporting the safety of this treatment. This suggests that other risk factors must still be identified. Baseline neurological deficits do appear to portend a higher risk of post-CED neurological deficit in our cohort. Factors such as the spatial relationship of the catheter tract with motor fibers, tumor characteristics related to aggressive disease, infusion rate, etc., are still being evaluated., $4,6,7,13$ Ultimately, comparing volumetric changes observed after CED to matched nonCED treated control patients will be vital in understanding the long-term results of CED.

Our results and interpretation are applicable only within the infusion parameters and disease continuum in this phase 1 clinical trial. The results are also limited by the small sample size and low statistical power of this study. As volumes of infusion or disease status changes, volumetric deformations and clinical tolerance may not be consistent with our results.

\section{Conclusions}

Our findings demonstrate that CED infusions into the brainstem of children with DIPG cause anatomical deformation. Specifically, increases in the brainstem volume and attenuation of the fourth ventricle with resultant ventriculomegaly occur with some correlation to infusion volume. Our findings are expected to contribute to more accurate interpretations of posttreatment MR scans. The results support a heightened awareness for hydrocephalus in children undergoing this therapeutic strategy. However, CED caused no symptomatic hydrocephalus in this cohort, and no patients required shunt placement. It remains uncertain if different infusion parameters will follow similar trends.

\section{References}

1. Anderson RCE, Kennedy B, Yanes CL, Garvin J, Needle M, Canoll P, et al: Convection-enhanced delivery of topotecan into diffuse intrinsic brainstem tumors in children. J Neurosurg Pediatr 11:289-295, 2013 
2. Bobo RH, Laske DW, Akbasak A, Morrison PF, Dedrick RL, Oldfield EH: Convection-enhanced delivery of macromolecules in the brain. Proc Natl Acad Sci U S A 91:2076-2080, 1994

3. Bredlau AL, Korones DN: Diffuse intrinsic pontine gliomas: treatments and controversies. Adv Cancer Res 121:235-259, 2014

4. Chen MY, Lonser RR, Morrison PF, Governale LS, Oldfield $\mathrm{EH}$ : Variables affecting convection-enhanced delivery to the striatum: a systematic examination of rate of infusion, cannula size, infusate concentration, and tissue-cannula sealing time. J Neurosurg 90:315-320, 1999

5. Essig H, Rana M, Kokemueller H, von See C, Ruecker M, Tavassol F, et al: Pre-operative planning for mandibular reconstruction - a full digital planning workflow resulting in a patient specific reconstruction. Head Neck Oncol 3:45, 2011

6. Ivasyk I, Morgenstern PF, Wembacher-Schroeder E, Souweidane MM: Influence of an intratumoral cyst on drug distribution by convection-enhanced delivery: case report. J Neurosurg Pediatr 20:256-260, 2017

7. Jagannathan J, Walbridge S, Butman JA, Oldfield EH, Lonser RR: Effect of ependymal and pial surfaces on convectionenhanced delivery. J Neurosurg 109:547-552, 2008

8. Kunwar S, Chang S, Westphal M, Vogelbaum M, Sampson J, Barnett G, et al: Phase III randomized trial of CED of IL13-PE38QQR vs Gliadel wafers for recurrent glioblastoma. Neuro Oncol 12:871-881, 2010

9. Lewis O, Woolley M, Johnson D, Rosser A, Barua NU, Bienemann AS, et al: Chronic, intermittent convectionenhanced delivery devices. J Neurosci Methods 259:47-56, 2016

10. Lonser RR, Walbridge S, Garmestani K, Butman JA, Walters HA, Vortmeyer AO, et al: Successful and safe perfusion of the primate brainstem: in vivo magnetic resonance imaging of macromolecular distribution during infusion. J Neurosurg 97:905-913, 2002

11. Luther N, Zhou Z, Zanzonico P, Cheung NK, Humm J, Edgar MA, et al: The potential of theragnostic ${ }^{124} \mathrm{I}-8 \mathrm{H} 9$ convectionenhanced delivery in diffuse intrinsic pontine glioma. Neuro Oncol 16:800-806, 2014

12. Luz M, Allen PC, Bringas J, Boiko C, Stockinger DE, Nikula KJ, et al: Intermittent convection-enhanced delivery of GDNF into rhesus monkey putamen: absence of local or cerebellar toxicity. Arch Toxicol 92:2353-2367, 2018

13. Morgenstern PF, Zhou Z, Wembacher-Schröder E, Cina V, Tsiouris AJ, Souweidane MM: Clinical tolerance of corticospinal tracts in convection-enhanced delivery to the brainstem. J Neurosurg 131:1812-1818, 2019

14. Murad GJA, Walbridge S, Morrison PF, Szerlip N, Butman JA, Oldfield EH, et al: Image-guided convection-enhanced delivery of gemcitabine to the brainstem. J Neurosurg 106:351-356, 2007

15. Occhiogrosso G, Edgar MA, Sandberg DI, Souweidane MM: Prolonged convection-enhanced delivery into the rat brainstem. Neurosurgery 52:388-394, 2003

16. Richardson RM, Gimenez F, Salegio EA, Su X, Bringas J, Berger MS, et al: T2 imaging in monitoring of intraparenchymal real-time convection-enhanced delivery. Neurosurgery 69:154-163, 2011

17. Sampson JH, Brady M, Raghavan R, Mehta AI, Friedman $\mathrm{AH}$, Reardon DA, et al: Colocalization of gadolinium-diethylene triamine pentaacetic acid with high-molecular-weight molecules after intracerebral convection-enhanced delivery in humans. Neurosurgery 69:668-676, 2011
18. Sampson JH, Brady ML, Petry NA, Croteau D, Friedman $\mathrm{AH}$, Friedman HS, et al: Intracerebral infusate distribution by convection-enhanced delivery in humans with malignant gliomas: descriptive effects of target anatomy and catheter positioning. Neurosurgery 60 (2 Suppl 1):ONS89-ONS99, 2007

19. Singleton WGB, Bienemann AS, Woolley M, Johnson D, Lewis $\mathrm{O}, \mathrm{Wyatt} \mathrm{MJ}$, et al: The distribution, clearance, and brainstem toxicity of panobinostat administered by convection-enhanced delivery. J Neurosurg Pediatr 22:288-296, 2018

20. Souweidane MM, Kramer K, Pandit-Taskar N, Zhou Z, Haque S, Zanzonico P, et al: Convection-enhanced delivery for diffuse intrinsic pontine glioma: a single-centre, doseescalation, phase 1 trial. Lancet Oncol 19:1040-1050, 2018

21. Valles F, Fiandaca MS, Bringas J, Dickinson P, LeCouteur R, Higgins R, et al: Anatomic compression caused by highvolume convection-enhanced delivery to the brain. Neurosurgery 65:579-586, 2009

22. Varenika V, Dickinson P, Bringas J, LeCouteur R, Higgins R, Park J, et al: Detection of infusate leakage in the brain using real-time imaging of convection-enhanced delivery. J Neurosurg 109:874-880, 2008

23. Wagner MEH, Gellrich NC, Friese KI, Becker M, Wolter FE, Lichtenstein JT, et al: Model-based segmentation in orbital volume measurement with cone beam computed tomography and evaluation against current concepts. Int J CARS 11:1-9, 2016

24. Yang X, Saito R, Nakamura T, Zhang R, Sonoda Y, Kumabe T, et al: Peri-tumoral leakage during intra-tumoral convection-enhanced delivery has implications for efficacy of peri-tumoral infusion before removal of tumor. Drug Deliv 23:781-786, 2016

25. Zhan W, Rodriguez y Baena F, Dini D: Effect of tissue permeability and drug diffusion anisotropy on convectionenhanced delivery. Drug Deliv 26:773-781, 2019

26. Zhou Z, Singh R, Souweidane MM: Convection-enhanced delivery for diffuse intrinsic pontine glioma treatment. Curr Neuropharmacol 15:116-128, 2017

\section{Disclosures}

Eva Wembacher-Schroeder, Rowena Thomson, and Elizabeth Vasconcellos are employees of Brainlab.

\section{Author Contributions}

Conception and design: Souweidane, Bander. Acquisition of data: all authors. Analysis and interpretation of data: Souweidane, Bander, Tizi, Wembacher-Schroder, Thomson, Vasconcellos. Drafting the article: Souweidane, Bander, Tizi, WembacherSchroder, Thomson. Critically revising the article: Souweidane, Bander, Wembacher-Schroder, Thomson. Reviewed submitted version of manuscript: Souweidane, Bander, WembacherSchroder, Thomson. Approved the final version of the manuscript on behalf of all authors: Souweidane. Statistical analysis: Souweidane, Bander. Administrative/technical/material support: Souweidane, Donzelli. Study supervision: Souweidane.

\section{Correspondence}

Mark M. Souweidane: Cornell University-Weill Medical College, New York, NY.mmsouwei@med.cornell.edu. 